\section{Transplante Simultâneo de Pâncreas-Rim em Portador de Diabetes Mellitus Tipo 1 com Insuficiência Renal Crônica. Experiência Inicial do Hospital Angelina Caron}

\begin{abstract}
RESUMO
O transplante pancreático é a melhor opção para substituir a função endócrina da glândula em pacientes com diabetes do tipo 1 (DM1). Ao final de 2001, já haviam sido notificados mais de 17.000 transplantes de pâncreas ao International Pancreas Transplant Registry. Na atualidade o transplante reno-pancreático simultâneo é o melhor tratamento para pacientes com DM1 e insuficiência renal crônica. Neste estudo são apresentados os resultados do transplante simultâneo de rim-pâncreas (SRP) realizados em um centro brasileiro. Métodos. De 01/2001 a 06/2002, 12 pacientes com DM1 e insuficiência renal associada foram submetidos a transplante SRP. A terapia imunossupressora consistiu de tacrolimus, micofenolato mofetil, esteróides e terapia de indução com basiliximab. Resultados. No seguimento de 5,7 meses (1-18), as taxas de sucesso para pâncreas e rim foram respectivamente de $75 \%$ e $83 \%$. A sobrevida dos pacientes foi de $83 \%$. Ocorreram algumas complicações sérias, como trombose de 3 pâncreas (25\%) e 2 rins (16\%) e 2 fístulas ureterais (16\%), porém nenhuma delas ocasionou óbito de qualquer paciente. Não ocorreu nenhum episódio de rejeição nos pacientes transplantados. Todos os doentes com enxertos funcionantes apresentam-se normoglicêmicos sem necessidade de insulina. Conclusões. Estes resultados demonstram que o SRP é um tratamento seguro e eficaz no manejo do doente diabético com insuficiência renal associada. (Arq Bras Endocrinol Metab 2003;47/3:243-247)
\end{abstract}

Descritores: Transplante pancreático; Diabetes; Insulina; Insuficiência renal

\section{ABSTRACT}

Simultaneous Pancreas-Kidney Transplant in Patients with Type 1 Diabetes Mellitus and Chronic Renal Failure. Initial Experience of Hospital Angelina Caron.

Pancreatic transplant is the best method for replacing the endocrine function of the gland in patients with type 1 diabetes (DM1). At the end of 2001, more than 17,000 pancreas transplants had been reported to the International Pancreas Transplant Registry. At present, simultaneous pancreaticorenal transplantation is the best treatment for DM1 patients with chronic renal failure. We present the results of simultaneous pancreas-kidney (SPK) transplantation performed in a Brazilian center. Methods. From $01 / 2001$ to 06/2002, 12 patients with DM1 and associated renal failure received a SPK. Baseline immunosuppression consisted of tacrolimus, mycophenolate mofetil, steroids and induction therapy with basiliximab. Results. With a mean follow-up of 5.7 months (range: 1-18), pancreas and kidney successful rates were respectively $75 \%$ and $83 \%$. Survival rate was $83 \%$. There were some major complications including 3 pancreas $(25 \%)$ and 2 kidney (16\%) venous thrombosis and 2 ureteral fistula (16\%), but none related to patient death. No episode of rejection occurred in any of the transplanted patients. All patients with successful grafts are insulin-free since transplantation. Conclusions. These data show that SPK is a safe and effective treatment in the management of DM1 patients with associated renal failure. (Arq Bras Endocrinol Metab 2003;47/3:243-247)

Keywords: Pancreatic transplantation; Diabetes; Insulin; Renal failure

\section{artigo original}

\author{
João Eduardo Nicoluzzi \\ Carlos W. Marmanillo \\ João Carlos D. Repka
}

Programa de Transplante

Hepático e Pancreático, Hospital

Angelina Caron, Campina

Grande do Sul, PR.
Recebido em 19/07/02

Revisado em 11/04/03

Aceito em 16/05/03 
E STIMA-SE QUE 0,3\% DA POPUlaÇão mundial seja acometida por diabetes mellitus tipo l (DMI) (1). Esse grande contingente de pacientes vem assistindo ao grande progresso no controle medicamentoso e de suporte da doença que, por muitos anos, ceifava vidas rapidamente em quadros de cetoacidose. A descoberta da insulina, entretanto, não significou a cura, mas apenas um controle parcial da doença, visto que, apesar dos múltiplos regimes terapêuticos disponíveis, as complicações secundárias da endocrinopatia podem apenas ser postergadas, mas não evitadas (2-4). Além disso, os pacientes são submetidos, continuamente, a um regime alimentar restrito.

Na década de 60, o transplante de pâncreas (TP) e o transplante de ilhotas pancreáticas eram estudados como uma nova solução para o diabetes. O primeiro transplante de pâncreas foi realizado em $1966 \mathrm{e}$, até outubro de 2001, mais de 17.000 transplantes pancreáticos haviam sido feitos em todo o mundo (5). A maior parte deles aconteceu após 1988, devido aos melhores resultados trazidos pela associação de imunossupressão com a ciclosporina e a conservação do enxerto com a solução de Wisconsin.

No entanto, a tolerância ao alotransplante de pâncreas exige o tratamento imunossupressor permanente, para evitar a rejeição do enxerto. Os riscos infecciosos e neoplásicos trazidos por esse tratamento desautorizam o emprego do transplante pancreático nas fases iniciais do diabetes, a título de profilaxia das complicações secundárias. Restringem-se, assim, as indicações de transplante pancreático isolado (TPI) aos diabéticos não-urêmicos hiperlábeis, com diabetes de difícil controle, crises freqüentes de cetoacidose, retinopatia rapidamente progressiva, coma hipoglicêmico, além de complicações secundárias $(6,7)$. Entretanto, na maioria dos centros, o transplante de pâncreas tem sido indicado em associação ao rim (8).

Após transplante de pâncreas bem sucedido, observa-se normalização do metabolismo dos carboidratos (9). Por si só isso aprimora incontestavelmente a qualidade de vida do paciente, mas não constitui o objetivo principal do procedimento, pois o paciente estaria puramente trocando a terapia insulínica vitalícia pela terapia imunossupressora, também vitalícia, associada a seus riscos inerentes. O principal objetivo do transplante de pâncreas consiste em reverter ou bloquear as complicações diabéticas secundárias. Estas incluem nefropatia no rim transplantado, neuropatia periférica e autônomica, doença micro e macrovascular e retinopatia.

O objetivo deste artigo é relatar a experiência inicial de nosso grupo em pacientes DM l com IRC submetidos a transplante simultâneo de pâncreas-rim, no período de 1,5 ano de programa.

\section{MATERIAL E MÉTODOS}

O programa de TP no Paraná foi iniciado em Janeiro de 2001 no Hospital Angelina Caron, Campina Grande do Sul, região metropolitana de Curitiba. De Janeiro de 2001 a Junho de 2002 foram realizados 12 TP simultâneos a um transplante renal (SPR) em pacientes com DMl, insulino-dependentes, com insuficiência renal associada, em programa de diálise ou com clearance de creatinina abaixo de $20 \mathrm{ml} / \mathrm{min}$.

Em todos, foi utilizado enxerto pancreático-duodenal com implantação na fossa ilíaca direita, sendo que, nos dois primeiros casos, a drenagem exócrina foi feita em alça digestiva e nos demais pacientes na bexiga.

Os órgãos foram captados segundo técnica já previamente descrita (10). Foi utilizado IL de solução de Wisconsin para flush aórtico. Após a retirada, tanto pâncreas quanto rim foram acondicionados em $1 \mathrm{~L}$ de solução de Wisconsin. Antes do implante, o enxerto pancreático foi dissecado no back-table com utilização da bifurcação ilíaca do doador para unir em um único tronco arterial as artérias esplênica e mesentérica superior do enxerto. A veia porta do doador foi dissecada até a confluência meso-esplênica sem a necessidade de utilização de enxerto venoso. As linhas de grampeamento duodenal foram reforçadas com fio monofilamentar de polipropileno 3.0, assim como a linha de grampeamento mesentérico.

Os pacientes foram selecionados para transplante de acordo com compatibilidade sangüínea $\mathrm{ABO}$ e teste de microlinfotoxicidade (cross-match) negativo. O TP foi feito com implantação nos vasos ilíacos comuns direitos com anastomose término-lateral. $\mathrm{O}$ enxerto renal foi implantado na fossa ilíaca esquerda com anastomose também do tipo término-lateral entre os vasos renais e ilíacos. A profilaxia antibiótica consistiu de doses pré, per e 3 doses pós-operatórias de cefazolina (lg IV). Todos os pacientes receberam dose diária de sulfametoxazol-trimetropin durante pelo menos três meses para profilaxia do Pneumocystis carinae. A profilaxia antifúngica consistiu de fluconazol via oral $150 \mathrm{mg} /$ dia por $2-3$ meses.

Para a imunossupressão primária foram utilizados tacrolimus, micofenolato mofetil e esteróides. Todos foram também submetidos à imunossupressão de indução com basiliximab $20 \mathrm{mg}$ IV no per-operatório e outra dose no quarto dia pós-operatório. $\mathrm{O}$ tacrolimus foi iniciado 4 horas após o transplante na dose de 0,1 a $0,2 \mathrm{mg} / \mathrm{kg}$ oral dividida em duas tomadas. O nível sérico desejado era entre $15-20 \mathrm{ng} / \mathrm{mL}$ nos primeiros três meses. Também 4 horas após o transplante, iniciou-se o micofenolato mofetil $2 \mathrm{~g} /$ dia em 2- 
4 doses. A dose foi reduzida nos casos de intolerância gastrointestinal ou leucopenia $\left(<3.000 / \mathrm{mm}^{3}\right)$. A corticoterapia foi feita IV com metilprednisolona $500 \mathrm{mg}$ em bolo no per-operatório, seguida de $250 \mathrm{mg}$ no pós e paulatinamente reduzida até $30 \mathrm{mg} /$ dia de prednisona oral do sétimo pós-operatório. Os resultados dos pacientes avaliados neste estudo são apresentados em médias e desvio-padrão.

\section{RESULTADOS}

Em 18 meses de programa, foram realizados 12 SPR, sendo 5 casos no primeiro ano e 7 nos últimos 6 meses. O grupo estudado era composto de 7 homens e 5 mulheres com idade média de 31,3 anos e com tempo médio de diabetes pré-transplante de 16 anos. Na época do transplante, apenas um paciente ainda não realizava sessões de diálise (clearance de creatinina $<20 \mathrm{ml} / \mathrm{min}$ ), os 11 restantes ( 10 hemodiálise, 1 diálise peritoneal) apresentavam tempo médio de duração de diálise de 23,1 meses.

O tempo médio de isquemia fria para o pâncreas foi de $8,5 \mathrm{~h}$ e para o rim de $10,5 \mathrm{~h}$. Todos os receptores apresentavam sorologia positiva para CMV, por este motivo não foi realizada profilaxia anti-viral.

As taxas de sobrevida dos pacientes e de sucesso dos enxertos renal e pancreático foram respectivamente de $83 \%, 83 \%$ e $75 \%$, com tempo médio de acompanhamento de 5,7 meses. Quanto ao enxerto renal, 9 pacientes apresentaram função imediata. Nos outros três casos, um paciente apresentou necrose tubular aguda, com normalização da função após duas semanas, e os outros dois perderam o enxerto por trombose da veia renal. Dois pacientes evoluíram para óbito: um deles apresentou episódio severo de hipotensão ortostática 2 semanas após a cirurgia, com perda tanto do enxerto pancreático quanto renal, evoluindo para óbito após dois meses por foco séptico abdominal; o outro ocorreu em paciente com ambos os enxertos funcionantes, um mês após a cirurgia por broncoaspiração. Quanto ao enxerto pancreático, todos os pacientes apresentaram normalização imediata da glicemia sem necessidade posterior de insulinoterapia. No seguimento, três pacientes evoluíram com perda do enxerto pancreático por trombose precoce, sendo em dois casos ainda na primeira semana e no outro na segunda semana, associado a episódio severo de hipotensão. Todos os 8 pacientes que estão atualmente com enxertos pancreáticos funcionantes apresentam-se normoglicêmicos sem necessidade de insulina ou de hipoglicemiantes orais. Nenhum deles segue qualquer tipo de regime ou restrição alimentar desde a alta hospitalar.

O tempo médio de internamento foi de 16,8 dias, sendo que $30 \%$ dos pacientes obtiveram alta hospitalar em 6 dias. Até o momento, nenhum paciente apresentou qualquer episódio de rejeição aguda. Quanto a outras complicações, 10 pacientes apresentaram infecções do trato urinário, sendo recidivantes em três. Ainda dois pacientes apresentaram broncopneumonia e outros dois infecções por CMV, que responderam ao tratamento com ganciclovir. Dois doentes apresentaram fístulas urinárias precoces e outros três sangramento pós-operatório com necessidade de reoperação. Não ocorreu nenhum óbito ou perda de enxertos devido a complicações cirúrgicas. Outros três pacientes apresentaram hematúria cáustica associada à drenagem exócrina pancreática do tipo vesical, tratadas com sucesso com instilação da bexiga durante 2 semanas com soro fisiológico frio.

\section{DISCUSSÃO}

Os resultados do TP melhoraram nos últimos anos, a ponto de alcançarem as boas taxas de sobrevida de enxerto observadas em transplantes de outros órgãos sólidos. Também, o TP foi influenciado beneficamente pela imunossupressão com a ciclosporina e a conservação com a solução da Universidade de Wisconsin ${ }^{\circledR}$.

A nefropatia recidivante nos pacientes diabéticos que recebem um transplante isolado de rim vem sendo uma preocupação constante para os cirurgiões de transplante. Em geral, terão que transcorrer vários anos antes dessa recidiva tornar-se clinicamente significativa, porém a evidência histológica de nefropatia diabética foi demonstrada no período pós-operatório imediato em receptores diabéticos de transplantes renais. Em acordo com essa opinião, o grupo de Estocolmo demonstrou que, nos pacientes submetidos a transplantes pancreático e renal combinados, as biópsias do enxerto renal obtidas de 2 a 4 anos após o transplante não mostravam qualquer evidência microscópica de nefropatia diabética recidivante $(11,12)$. Outros grupos demonstraram um efeito benéfico semelhante do transplante de pâncreas sobre a nefropatia diabética $(13,14)$. Em outro estudo, com seguimento dos pacientes por 10 anos pós-transplante, foi demonstrado que pacientes diabéticos com insuficiência renal crônica que receberam unicamente enxerto renal a sobrevida foi de $37 \%$ versus $60 \%$ em relação aos pacientes submetidos a SPR (15).

Muitos pacientes submetidos a um TP indicam subjetivamente uma certa melhora na neuropatia pe- 
riférica (16). Entretanto, nos casos de transplante combinado rim-pâncreas, é difícil estabelecer a diferença entre as melhoras na neuropatia urêmica versus diabética. Certos estudos demonstraram melhora na velocidade de condução nervosa tanto motora quanto sensorial após o transplante de pâncreas (17). Outros estudos também demonstraram melhora subjetiva na neuropatia autônoma, incluindo a gastroparesia, em um grupo de receptores de transplante combinado rim-pâncreas, em comparação com um grupo de pacientes diabéticos que haviam recebido um transplante isolado de rim $(18,19)$. Com a utilização da termografia, das mensurações da tensão do oxigênio muscular e da determinação Doppler com laser, foi demonstrado efeito benéfico do transplante de pâncreas sobre a microcirculação (20). No entanto, não existem dados conclusivos capazes de demonstrar uma menor taxa de amputações e de úlceras diabéticas das extremidades inferiores nos pacientes submetidos a um transplante de pâncreas bem sucedido.

Os efeitos do transplante de pâncreas sobre a retinopatia diabética continuam sendo controversos (21). Os estudos iniciais do grupo de Minnesota demonstraram progressão da retinopatia diabética após o TP (22). Em contraste, outro estudo destinado a comparar vários parâmetros da retinopatia diabética nos pacientes com transplante combinado rim-pâncreas, versus receptores diabéticos de um transplante isolado de rim, demonstraram melhora significativa nas modificações globais no grupo com transplante combinado (23).

Anteriormente, notava-se a morbidade especialmente alta do TP ligada, sobretudo, à porção exócrina do pâncreas. Na série ora apresentada, a complicação predominante foi justamente o grande número de infecções do aparelho urinário. Este tipo de complicação ocorre, em algum momento, na quase totalidade dos pacientes, e é conseqüente à drenagem da secreção pancreática exócrina na bexiga (24). Ainda outras complicações relacionadas à drenagem vesical são acidose metabólica, uretrite e cistite. Para evitar estas complicações urológicas, existe uma tendência atual de drenagem do pâncreas em alça digestiva (25). Porém, a drenagem vesical, apesar de seus inconvenientes, é mais segura, pois uma fístula digestiva associada à drenagem entérica é geralmente fatal.

A rejeição aguda ao enxerto pancreático pode ocorrer isolada (18\%) ou simultaneamente à rejeição do enxerto renal, quando este está associado $(33 \%)(26,27)$. Nesta série, não foram observados episódios de rejeição, o que pode ser devido tanto ao esquema imunossupressor agressivo por nós utilizado, quanto ao tempo de seguimento dos pacientes, uma vez que a maioria dos episódios de rejeição ocorre no primeiro ano.

A trombose vascular tem incidência variável entre os diferentes serviços. Em algumas séries, ela ocorre em até $15 \%$ dos transplantes, dentro das primeiras 24 horas de pós-operatório $(28,29)$. Na casuística deste estudo, foi observado em três pacientes o que corresponde a $25 \%$ dos casos. Tais complicações foram relacionadas com a conservação do enxerto e com a qualidade das anastomoses vasculares.

Apesar da morbidade do TP, e do efeito global sobre as complicações diabéticas secundárias ser variável, vários estudos evidenciaram uma melhor qualidade de vida nos receptores de TP e até uma mudança da própria história natural da doença e da perspectiva de vida deste grupo de doentes, incluindo a observação de gestações bem-sucedidas após o transplante (30). Atualmente o SPR é a melhor alternativa para pacientes com DMl e com insuficiência renal crônica associada (31).

\section{REFERÊNCIAS}

1. Deckert T. Insulin-dependent diabetes mellitus and its complications. In: Groth CG, editor. Pancreatic transplantation. WB Saunders: Philadelphia, 1988:1-14.

2. Cretin N, Bhler L, Fournier B, Caulfield A, Oberholzer J, Mentha $G$, et al. Human islet allotransplantation: world experience and current status. Dig Surg 1998;59:263-8.

3. Margreiter R, Ofner D, Reinl E, et al. Pancreas transplantation - a critical appraisal. Transplant Proc 1992;24/2:383-6.

4. Ratner RE. Type 2 diabetes mellitus: the grand overview. Diabet Med 1998; 15 Suppl 4:S4-S7.

5. Gruessner AC, Sutherland DER. Analysis of United States (US) and non-US pancreas transplants reported to the United Network for Organ Sharing (UNOS) and the International Pancreas Registry (IPTR) as of October 2001. Clin Transpl 2001;41-72.

6. Sutherland DER, Gores PF, Farbey AC, et al. Evolution of kidney, pancreas, and islet transplantation for patients with diabetes at the University of Minnesota. Am J Surg 1993; 166:456-84.

7. Moudry-Munss KC, Gruessner A, Sutherland DER. International pancreas transplant registry report 1993. Transplant Proc 1994;26:407-11.

8. Reichard $P$, Bengt-Yngve N, Rosenqvist $U$. The effect of long-term intensified insulin treatment on the development of microvascular complication of diabetes mellitus. N Engl J Med 1993;329:304-9.

9. Landgraf R, Landgraf-Leurs MMC, Burg D, et al. Longterm follow-up of segmental pancreas transplantation in type 1 diabetes. Transplant Proc 1986; 18:1118. 
10. Boudjema K, Ellero B, Odhe M, et al. Techniques des prélèvements multiorganes. In: Encyclopedie MédicoChirugicale (ed.). Techniques chirugicales; Appareil digestif. Editions Techniques: Paris, 1993.

11. Bohman SO, Tyden G, Wilczek H, et al. Prevention of kidney graft diabetic nephropathy by pancreas transplantation in man. Diabetes 1985;34:306-8.

12. Bohman SO, Wilczek $H$, Jarenko $G$, et al. Recurrence of diabetic nephropathy in human renal allografts: A preliminary report of a biopsy study. Transplant Proc 1984; 16:649.

13. Bilous RW, Mauer SM, Sutherland DER, et al. The effects of pancreas transplantation on the glomerulus structure of renal allografts in patients with insulin-dependent diabetes. N Engl J Med 1989;321:80.

14. Sutherland DER. Effect of pancreas transplants on secondary complication of diabetes: Review of observations at a single institution. Transplant Proc 1992;24:859.

15. Tyden G, Tollemar J, Bolinder J. Combined pancreas and kidney transplantation improves survival in patients with end-stage diabetic nephropathy. Clin Transplant 2000; 14:505-8.

16. Solders G, Tyden G, Persson A, et al. Diabetic neuropathy fours years after pancreas transplantation. Transplant Proc 1992;24:856.

17. Naouri A, Martin X, Dubernard JM. Evolution of diabetic neuropathy after kidney pancreas transplantation. Transplant Proc 1992;24:875.

18. Gaber AO, Hathaway D, Cardoso S, et al. Improvement in autonomic and gastric function in pancreas-kidney versus kidney alone transplantation and the impact on quality of life. Transplantation 1991;57:816-22.

19. Murat A, Pouliquem B, Canturonich D, et al. Gastric emptying improvement after simultaneous segmental pancreas and kidney transplantation. Transplant Proc 1992;24:855

20. Alendroth D, Ilhmer VD, Landgra FR, et al. Are late diabetic complications reversible after pancreas transplantation? A new method of follow-up of microcirculatory changes. Transplant Proc 1987; 19:23.

21. Bandello F, Vigano C, Seachi A, et al. The influence of combined kidney-pancreas transplantation advanced diabetic retinopathy. Transplant Proc 1992;24:854.
22. Ramsy RC, Goetz FC, Sutherland DER, et al. Progression of diabetic retinopathy after pancreas transplantation for insulin-dependent diabetes mellitus. N Engl J Med 1988;318:208.

23. Wang $Q$, Klein $R$, Moss SE, et al. The influence of combined kidney-pancreas transplantation on the progression of diabetic retinopathy. Ophthalmology 1994;101:1071-6.

24. Castoldi R, Staudacher C, Ferrari G, et al. Early postoperative surgical complications: comparison of segmental duct-injected versus whole bladder-drained pancreas transplantation. Transplant Proc 1992;24:817-20.

25. Sollinger HWO, Sasaki TO, D'Alessandro AM, et al. Indications for enteric conversion after pancreas transplantation with bladder drainage. Surgery 1992;112:842-6.

26. Klassen DK, Hoen-Saric EW, Weir MR, et al. Isolated pancreas rejection in combined kidney pancreas transplantation. Transplantation 1996;61:974-7.

27. Kuo PC, Johnson LB, Schweitzer EJ, et al. Solitary pancreas allografts. The role of percutaneous biopsy and standardized histologic grading of rejection. Arch Surg 1997; 132:52-7.

28. Ozaki CF, Stratta RJ, Taylor RJ, et al. Surgical complications in solitary pancreas and combined pancreas-kidney transplantations. Am J Surg 1992;42:727-36.

29. Whelchel JD, Larse C, Olsen R, et al. Simultaneous pancreas kidney transplantation: Initial experience of the Emory University Transplant Service. Int Surg 1994;79:98102.

30. Calne RY, Brons IGM, Williams PF, et al. Successful pregnancy after paratopic segmental pancreas and kidney transplantation. BRMJ 1988;296:1709.

31. Dubernard JM, Tajra LC, Lefrançois $L$, et al. Pancreas Transplantation: results and indications. Diabetes Metab 1998;24:195-9.

\section{Endereço para correspondência:}

João Eduardo Nicoluzzi

Rua Santo Amaro, 118

80620-330 Curitiba, PR 